\title{
ACR: APPLICATION AWARE CACHE REPLACEMENT FOR SHARED CACHES IN MULTI-CORE SYSTEMS
}

\author{
Dr. Tripti S Warrier \\ Dept. of Electronics, Cochin University of Science and Technology (CUSAT), \\ Cochin, Kerala State, India
}

\begin{abstract}
Modern multi-core systems allow concurrent execution of different applications on a single chip. Such multicores handle the large bandwidth requirement from the processing cores by employing multiple levels of caches with one or two levels of private caches along with a shared last-level cache (LLC). In shared LLC, when applications with varying access behavior compete with each other for space, conventional single core cache replacement techniques can significantly degrade the system performance. In such scenarios, we need an efficient replacement policy for reducing the off-chip memory traffic as well as contention for the memory bandwidth.

This paper proposes a novel Application-aware Cache Replacement (ACR) policy for the shared LLC. ACR policy considers the memory access behavior of the applications during the process of victim selection to prevent victimizing a low access rate application by a high-access rate application. Itextcolor\{red\}\{ It dynamically tracks the maximum life-time of cache lines in shared LLC for each concurrent application and helps in efficient utilization of the cache space. Experimental evaluation of ACR policy for 4-core systems, with 16-way set associative 4MB LLC, using SPEC CPU 2000 and 2006 benchmark suites shows a geometric mean speed-up of $8.7 \%$ over the least recently used (LRU) replacement policy. We show that the ACR policy performs better than recently proposed thread-aware dynamic re-reference interval prediction (TA-DRRIP) and protecting distance based (PDP) techniques for various 2-core, 4-core and 8-core workloads.
\end{abstract}

Keywords: Multi-core system, Last Level Cache, Shared Cache Management, Application-aware, Misses per Kilo Instructions (MPKI), Replacement Policy.

Cite this Article: Dr. Tripti S Warrier, ACR: Application Aware Cache Replacement for Shared Caches in Multi-Core Systems, International Journal of Computer Engineering and Technology, 10(2), 2019, pp. 234-251.

http://iaeme.com/Home/issue/IJCET?Volume=10\&Issue=2 


\section{INTRODUCTION}

Modern multi-core systems support multiple levels of caches to improve the overall performance of the system by reducing the memory accesses. The LLC in such multi-core systems is shared among concurrent applications. Efficiency of the utilization of the LLC space depends on the implementation of LLC management policy. An efficient implementation of LLC management policy leads to reduction in off-chip memory traffic which has a direct impact on the performance of the system. The most important component of cache management policy is the cache replacement policy. An ideal replacement policy will victimize cache lines that will be accessed farthest in future and retain the cache lines having high temporal locality [1]. However, all practical cache replacement policies take victim selection decision by predicting a cache line that is going to be re-referenced farthest in future $[2,10]$. The effectiveness of such replacement policies depends on the accuracy of the prediction.

Traditionally, LLCs in multi-core system employ the binary search approach of the Least Recently Used (LRU) replacement policy [2] due to its ease of implementation. Figure 1 shows the performance of individual applications from multiprogrammed workloads in a 4core system with an un-managed shared LLC ( $4 \mathrm{MB}$ - 1MB per-core is considered for the experiments) employing LRU replacement policy. Performance is measured in terms of the instructions per clock cycle $(I P C)$ of the individual application. The plot shows the normalized $I P C$ of each application when it is running in a shared environment with respect to the case when it is running alone. Lower the bar, higher is the performance degradation experienced by an application due to sharing. The figure shows that, the interference in the LLC between the cores that compete for LLC space lead to severe decline in the system performance. For example, the applications in workload 3 experience a degradation of around $29 \%$ ( for $h 264 r e f$ ) to $40 \%$ ( for $m c f$ ). Furthermore, it can be seen that the applications bzip2 and h264ref that are common to workload1, workload 2 and workload3 experience varying degree of performance degradation in different workloads even though the underlying system is the same. The variation in performance of these applications is due to the difference in working sets (Amount of memory that an application requires in a given time) of applications that are co-scheduled with bzip2 and h264ref in different workloads. These working sets can interfere with each other and affect the miss rates at the shared LLC. An application with high misses per kilo instruction (MPKI) is responsible for inserting large number of cache lines into the shared LLC. A high MPKI application with its high access rate will flush the cache lines of a low MPKI application if the shared LLC maintains a common eviction priority chain for all the scheduled applications. When multiple applications are scheduled simultaneously, the performance of all the applications can typically degrade due to the contention for space in the LLC among the concurrently executing applications. The replacement policy used, does not (i) distinguish between various applications that share the LLC; (ii) understand the memory requirements of those applications. This discussion shows that there is a need for an application aware cache replacement policy for the shared LLC in multi-core systems that maintains an This discussion shows that there is a need for an application aware cache replacement policy for the shared LLC in multi-core systems that maintains an eviction priority chain based on the requirement of the applications.

eviction priority chain based on the requirement of the applications. 


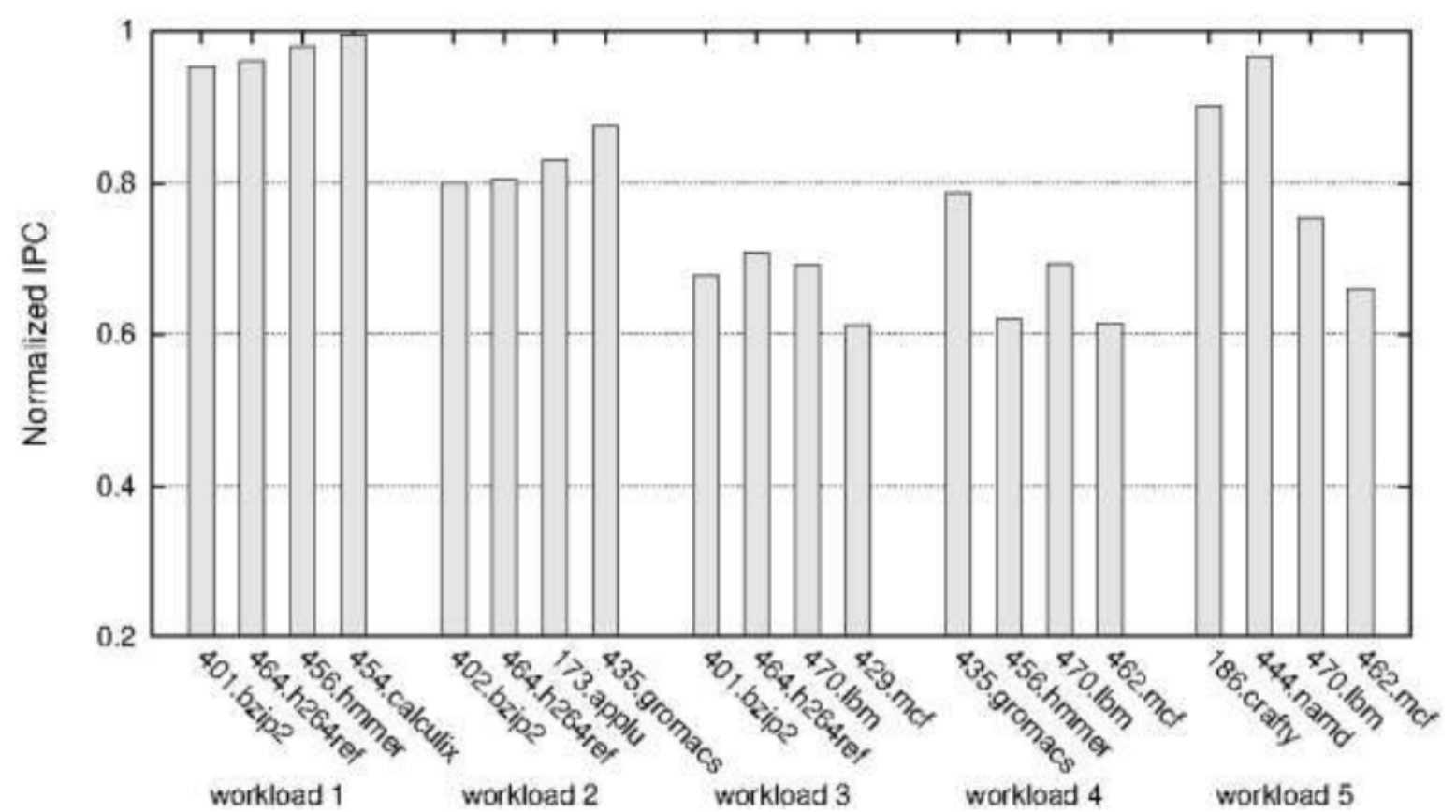

Figure 1 Degradation in the performance of individual applications when the applications are coscheduled in a multi-core system with a shared 4MB LLC. The bars show the normalized IPC of each application running in a shared environment with respect to the $I P C$ when it is running alone.

Adopting a replacement policy that is aware of the characteristics of the application memory accesses in the LLC can lead to better management of the LLC space. Many recent cache replacement policies proposed for multicore LLCs $[3,4,5,6]$ try to meet these requirements by depending on the data access patterns of the specific workloads. An ideal replacement policy must allow sufficient time to the cache lines in a cache and provide them with opportunity to be reused. Furthermore, it must not retain the cache lines for too long and prevent the cache pollution. Figure 2 shows the distribution of LLC accesses for various benchmarks, based on the reuse distance. The reuse distance of a cache line is measured in terms of the number of accesses to the set between consecutive accesses to the cache line. We consider a fixed size LLC (i.e., 1MB 16-way set associative cache). Each access from the LLC access trace of an application is put into one of the five bins based on the reuse distance of the cache line that is being accessed. These five bins correspond to the following mutually exclusive integral ranges for the reuse distance values: [0-7]; [8-15]; [16-31]; [32-63] and $[0 \geq 64]$ (implies no reuse or reuse distance larger than 63). Figure 2 provides the percentage of cache accesses in each of these categories. For the application gzip, $78 \%$ of the cache lines have a reuse distance of less than 8 . Hence, if all the cache lines of gzip were provided with maximum time of 8 accesses to the set (not worrying about associativity or replacement) in the cache then $78 \%$ of the LLC accesses can be hit. Since this behavior of cache accesses depends only on the application's reuse characteristic. There are several applications such as gzip, vpr, twolf, gromacs, hmmer, and h264ref that experience significant cache hits if the maximum allowable time of the cache lines in the LLC is restricted to 15 accesses to a cache set. For these applications the reuse distance of majority of the cache lines is limited to a particular value. Similarly there is another set of applications such as swim, mcf, libquantum, and $\mathrm{lbm}$ that has zero or very few accesses to the cache with in the bin size of less than 64 . 


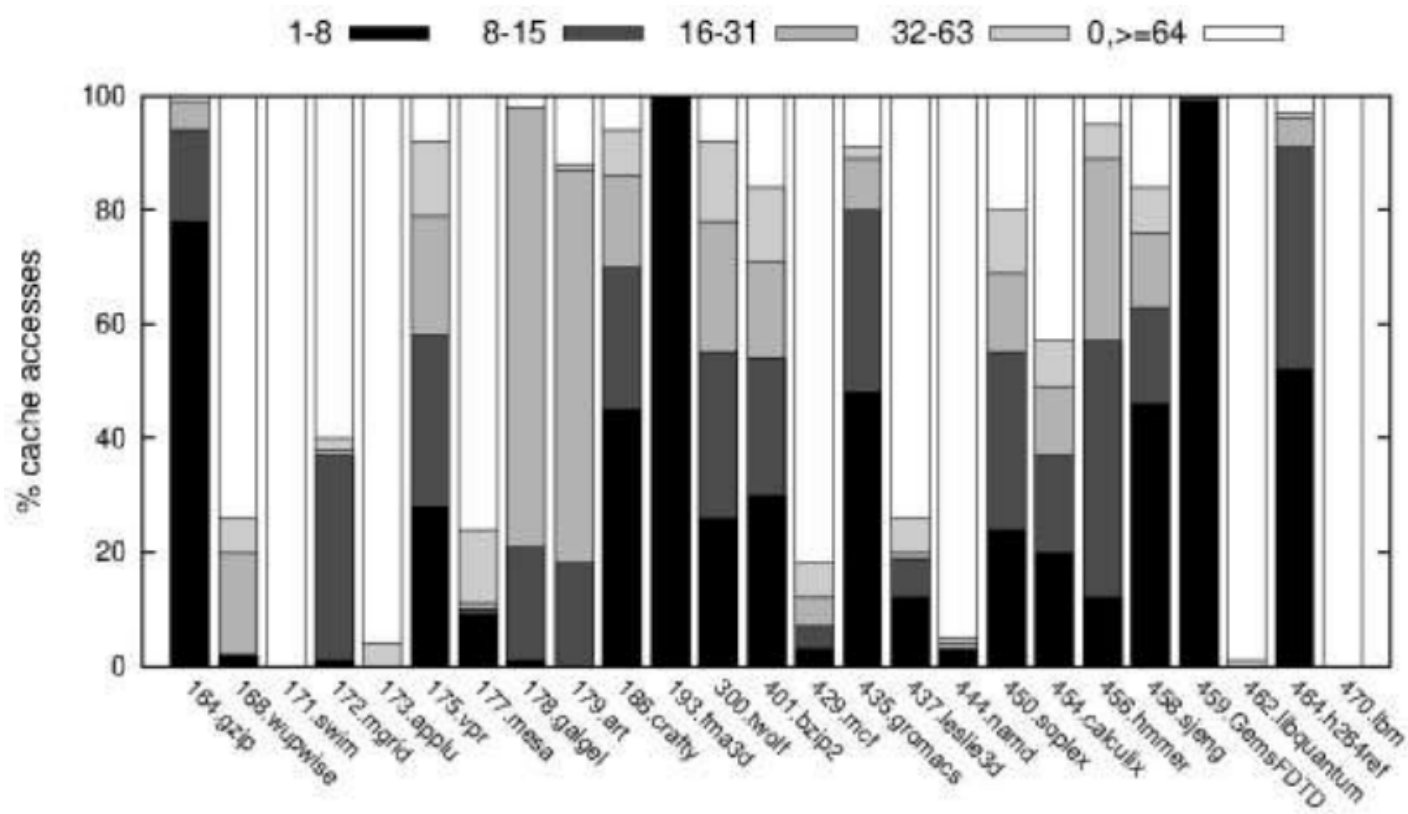

Figure 2 LLC accesses for single core SPEC benchmarks with reuse distance measured based on access count.

This shows that such applications may have very few cache hits even if we retain the cache lines from these applications for much longer time (of 63 accesses to the set) in the cache. For this set of applications, the reuse distance is so large that keeping the cache lines for long may pollute the cache and penalize other co-scheduled applications. This study shows that the reuse distance of the cache lines from an application can provides some hints on the maximum life-time of cache lines in the cache and can be used to manage LLC.

This article proposes an Application-aware Cache Replacement (ACR) policy (Part of this work has been published in [7]) that exploits reuse distances in cache accesses to capture the access behavior of the applications sharing the LLC. Our study of reuse distances at LLC for cache lines that belong to the applications from SPEC CPU 2000 and 2006 benchmark suites [8] shows that each application has different reuse distance distribution based on its access pattern. The maximum reuse distance of an application is the maximum life-time for any of its cache lines in the LLC. If a cache line is allowed its maximum time (termed hit-gap in this paper) in the LLC, most of the re-references to this cache line may result in hits. The eviction of this cache line after its allotted time by the application or any other co-scheduled application will not penalize the application. We compare the ACR policy with the LRU policy, thread-aware dynamic RRIP (TA-DRRIP) [4] policy and protecting distance-based replacement policy (PDP) [9] using SPEC CPU 2000 and 2006 benchmarks. Our performance studies show that ACR policy improves cache performance over these replacement policies. The major contribution of the work is that the proposed replacement technique works well for workloads with both LRU-friendly and thrashing access patterns as opposed to the TADRRIP, which is not the best replacement technique for LRU-friendly workloads. Also the proposed technique gives better speedup in contrast to a reuse distance based policy, i.e., PDP, with much simpler implementation complexity.

The rest of the paper is organized as follows: Section 2 provides review of the previous work, followed by details of the proposed technique in Section 3. Section 4 describes the experimental setup and the results are analysed in Section 5. and Section 6 gives the concluding remarks. 


\section{RELATEDWORK}

As effectiveness of LLC is crucial for multi-core system performance, several cache eviction policies for LLC have been proposed in the literature for both single-core and multi-core systems. Discussion in this section is restricted to techniques that are relevant to the proposed technique.

Counter-based replacement technique [10] for LLC in unicore predicts access interval for cache lines using a per-cache line access counters. A cache line whose counter value exceeds a given threshold is selected as victim during eviction. It also identifies cache lines that are dead on access and bypasses the LLC. Another fine-grained approach namely, adaptive timekeeping replacement policy [5] employs the operating system to assign the life-time to cache lines in the LLC.

Reuse distance information of cache lines can reveal the temporal locality of the data used by the applications. Reuse distance based replacement policies [11, 12, 13] exploit reuse distance during victim selection for unicores. Reuse distance predictor based on PC is used to drive the replacement policy in [11,12]. Correlation between the access patterns of instructions and the temporal characteristic of cache lines is exploited to predict the reuse distance in [13]. Protected distance based policy (PDP) [9] protects the cache lines in the cache for certain number of accesses to the set called the protection distance (PD). The PD is dynamically computed using a hit rate based model which is implemented using a sampler structure and special-purpose processor. Next-use cache (NUCache) [14] utilizes the relation of the access pattern with PC to maximize the hits at the LLC. NUCache provides extra time for cache lines of an application being brought by special PCs.

Thread-aware replacement techniques $[3,4,15]$ predict future re-reference of cache lines and try to save only those cache lines of the application that will be re-referenced in the near future. More recently, the performance of the tree based PseudoLRU for single core LLC has been improved by modifying the position of insertion and promotion [16]. This adaptive PseudoLRU insertion and promotion algorithm provides performance similar to DRRIP and PDP with much lower overhead.

Multi-core cache shared cache partitioning policy, utility-based cache partitioning (UCP) [17], partitions the ways in the sets based on the utility of the application. UCP only tries to maximize the hits but cannot provide any guarantee of cache hits for re-references. Promotion/Insertion Pseudo-Partitioning (PIPP) [6] also monitors application utility but does a pseudo partitioning with the help of dynamic promotion and insertion policies. Thrasher caging [18] identifies thrashing applications that degrade the performance of multicores and restricts the number of ways allocated to such applications. Thrasher caging prevents pollution of cache by thrashing application but does not handle interference among nonthrashing application as is done by the ACR policy. More recently, mechanism called Vantage [19] has been proposed which is an analytically model based cache partitioning that can be used along with other replacement policy. PriSM [20] proposes framework based on eviction probabilities to manage the cache occupancy of different cores at cache line granularity. The eviction probability distribution, computed by the allocation policy of the PriSM is used to identify a victim core id. 


\section{ACR : APPLICATION-AWARE CACHE REPLACEMENT POLICY}

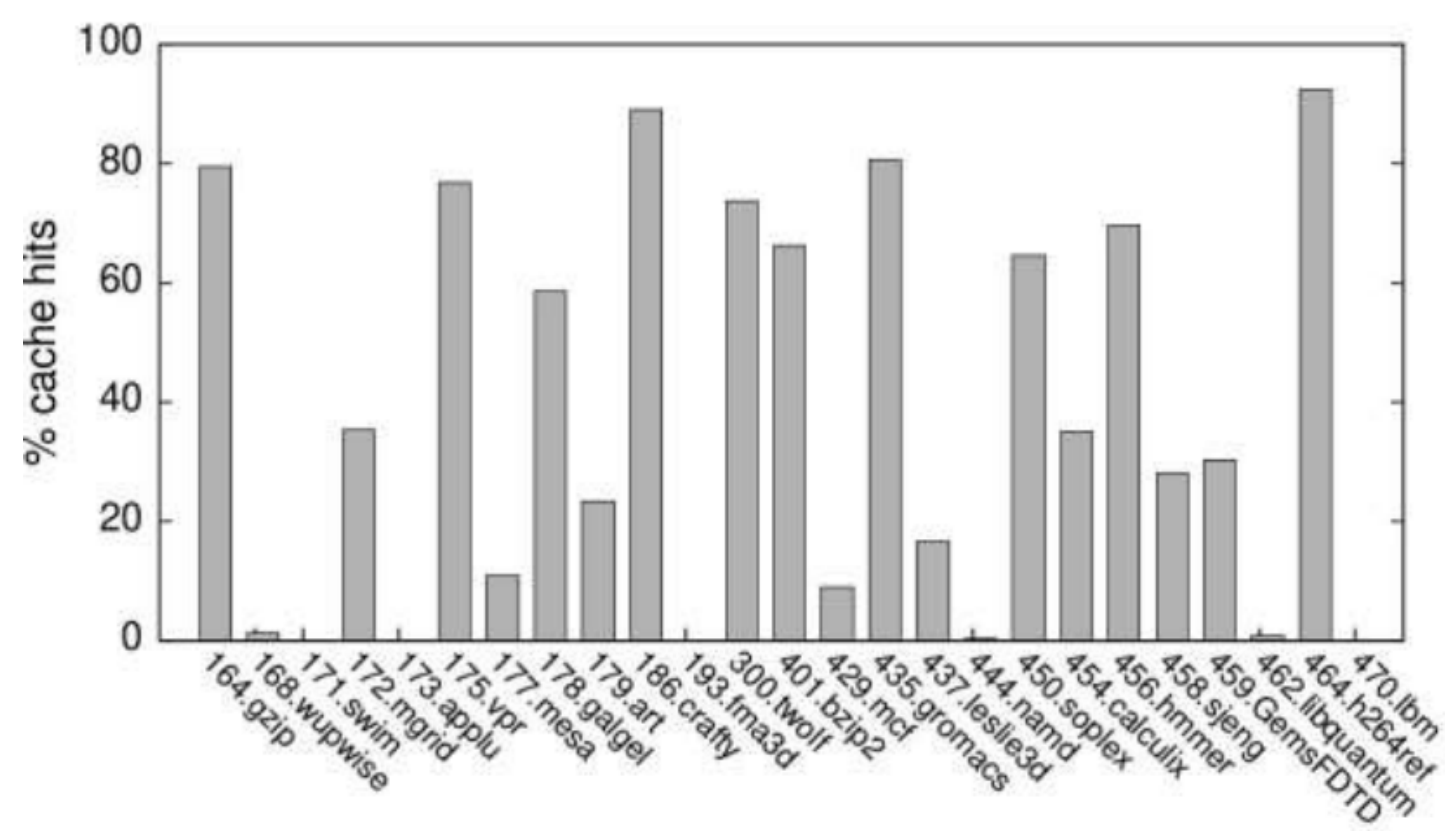

Figure 3 LLC accesses for single core SPEC benchmarks with reuse distance measured based on access count.

The reuse distances of cache lines can be used by the replacement policy to control the life-time of the cache lines in the cache. The life-time thus determined can ensure hits to most of the re-references to the LLC. The maximum time of a cache line in the LLC, decided based on the reuse distance, is termed as hit-gap. Allotting a particular hit-gap $r$ to an application will ensure all cache lines to be present in the cache for a period of $r$. This can ensure hits for all the cache references that have a reuse distance of $r$. To verify the inference obtained from the oracle study in Figure 2, we implement a replacement policy with hit-gap as 15 for cache lines in the LLC. The victim during eviction would be a cache line with least time left in the cache. We obtain the amount of time left for a cache line in cache by comparing the current life-time of the cache line in cache (measured using accesses to the cache set) and hit-gap. In case there are multiple cache lines with same time remaining in a cache set, leftmost cache line among these multiple cache lines is selected as victim. Figure 3 depicts the hit rate of individual applications for the above mentioned LLC size and associativity, with the new replacement policy. The LLC hit rate of gzip is around $80 \%$ while that predicted from Figure 2 is $94 \%$. It can be seen that the actual hit rate for the LLC using hit-gap of 15 follows the trend observed in Figure 2.

Based on the above observations, we propose an age-based Application-aware cache replacement (ACR) policy that uses the maximum reuse distances i.e. hit-gap to of the applications to find the victim during cache replacement. The maximum reuse distance of cache lines for the co-scheduled applications is tracked dynamically for each individual application. The use of maximum reuse distance as hit-gap will ensure that all the cache lines of an application are present in the cache for the allotted time. This can prevent early eviction of cache lines and retain them till the time of next use. A cache line, whose current life-time is greater than the current hit-gap of the corresponding application, can be considered as the victim during the eviction process. Eviction of such a cache line during a miss may not penalize the original application since the cache line was given its fair share of time in the 
cache. This process of limiting the maximum life of cache lines can also prevent the pollution at the LLC.

\subsection{Basic Concept}

Application-aware cache replacement (ACR) policy proposes two changes to the chain (Figure 4)

- Separate the eviction priority chains for individual applications: Figure 1 shows that the separation of eviction priority chains for different applications can reduce the interference of high miss rate application on other co-scheduled applications. Based on this the ACR policy updates the eviction priority of only those cache lines that belong to the referencing application in order to maintain separate eviction priority chains for each of the applications.

- Dynamically adjusting the length of different eviction priority chains: The ACR policy dynamically tracks the maximum reuse distance of an application for each interval and uses it as the predicted life-time or predicted hit-gap $(P H G)$ for the next interval. This hit-gap is used to control the length of individual eviction priority chain at run-time to make the ACR policy aware of the access pattern.

Further, we enhance the replacement policy to handle victim selection when there are multiple victim candidate. This is done by keeping tab on the order of access recency among the concurrent applications for individual sets. This will ensure that the proposed technique is aware of both reuse distance and access recency, resulting in support to both cache-friendly and scan access patterns.

The work proposes two separate implementations of ACR policy namely :

- access count based Application-aware Cache Replacement (aACR) policy : The aACR policy measures the reuse distance of a cache line based on the number of accesses to the cache set between consecutive accesses to the cache line.

- miss count based Application-aware Cache Replacement (mACR) policy : The mACR measures the reuse distance of a cache line based on the number of misses to the cache set between consecutive accesses to the cache line.

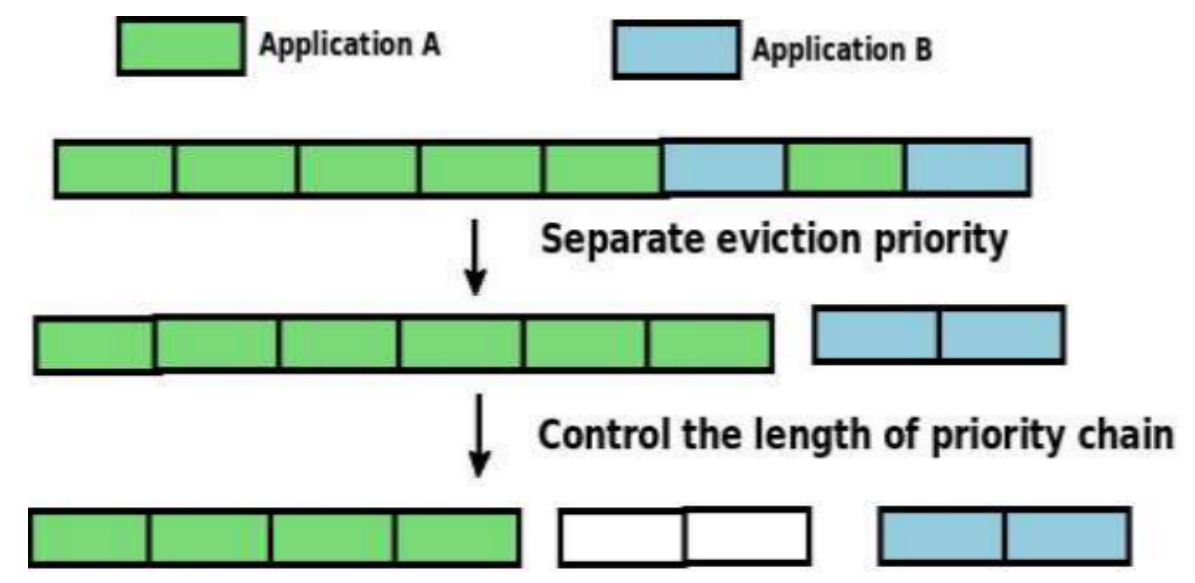

Figure 4: Changes proposed in the eviction priority chain. 


\subsection{Basic Implementation}

Each cache line has a $n$-bit saturating counter, called hit-gap counter (HG), that gives the priority or age of the cache lines in their corresponding cache set. The $H G$ counter of each cache line is modified based on the behaviour of the accessing application and is used to track of the amount of time spent by a cache line in the cache without an access. To prevent the inter-application interference in shared LLC, the $H G$ counter values is not modified by the behavior of other applications accessing LLC. Hence, unlike in LRU, cache access in the ACR policy can trigger the updation of the $H G$ counters of cache lines that belong to the accessing application only. This requires an additional $\log _{2} C$ bits per cache line to that indicates the associated core id, where $\mathrm{C}$ is the number of cores in the system. The $H G$ counter value of a cache line at any given time is an estimate of the cache line life-time in the cache after its last access. A cache line with the highest counter value is the oldest cache line without any access in the set. If an access is a hit, the $H G$ counter of the corresponding cache line is reset. The value of the $H G$ counter at the time of hit is the hit-gap of the cache line. When a new cache line is inserted, its $H G$ value is set to 0 .

For dynamically tracking the life-time (maximum hit-gap) of cache lines of the applications, two $n$-bit application-wise register, predicted-hit-gap (PHG) and shadow predicted-hit-gap ( $\mathrm{P} P H G$ ) are maintained. $P H G$ value holds the predicted life-time for current interval of the application and controls the length of eviction priority chain of the application. $s P H G$ holds the learned maximum hit-gap during the current interval (to be used as the predicted lifetime for the next interval). The total number of cache sets $\mathrm{s}$ is divided into $\mathrm{p}$ monitor sets and $\left(s^{-}-\mathrm{p}\right)$ normal sets. The predicted life-time for all the monitor sets is fixed to MAX $=2^{\mathrm{n}}-1$ (for $H G$-counter of width n-bit) to ensure the maximum possibility of hit-gap for cache lines in the monitor sets. The hit gaps of the monitor set and the normal sets are tracked and stored as $s P H G$ for each interval of $2 \mathrm{~m}$ misses (implemented using an $\mathrm{m}$-bit miss Counter) in the LLC. The PHG value is updated at the end of each interval (refer to Figure 5) based on the status of 1-bit hitFlag.

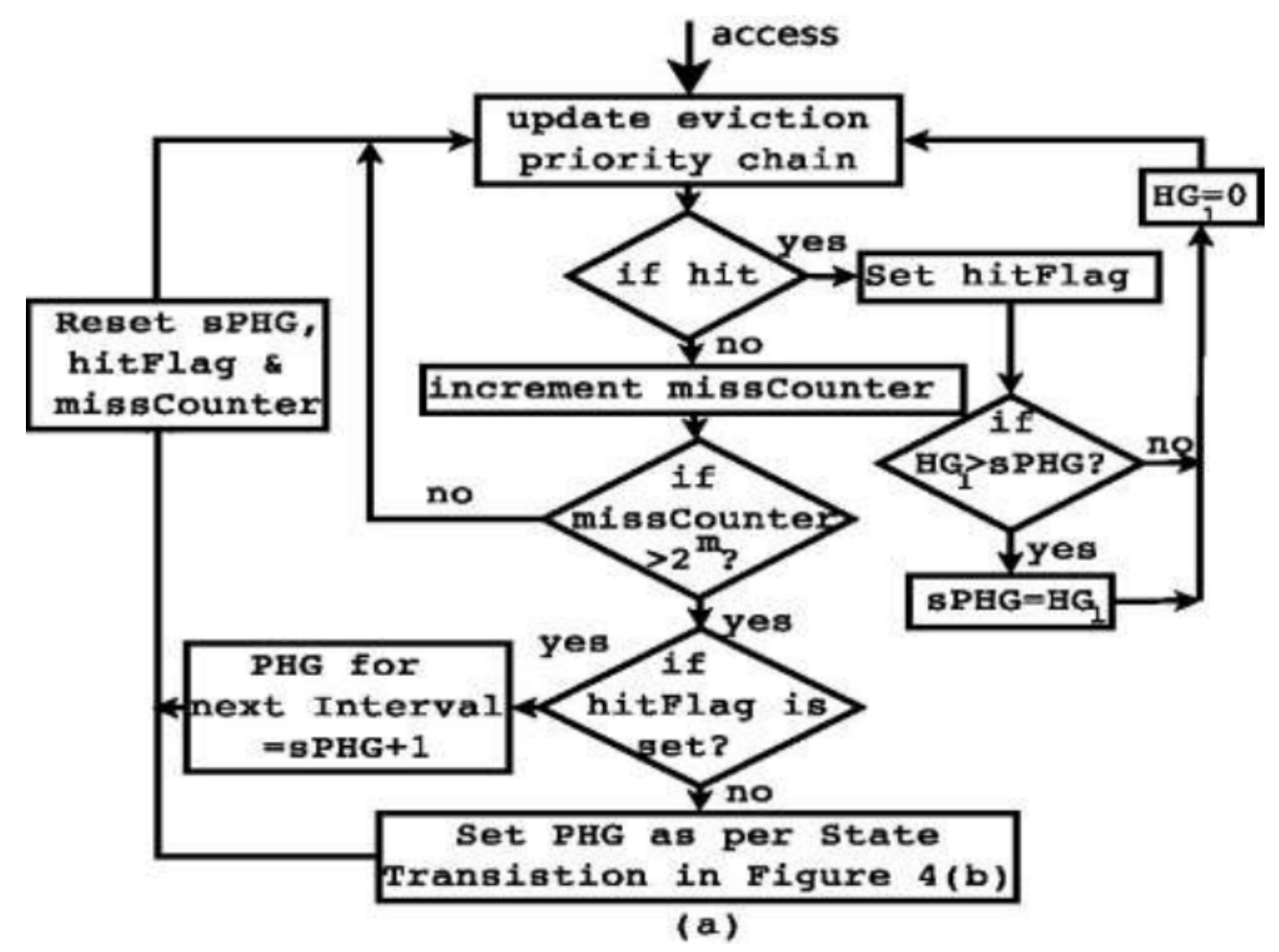

Figure 5: Flowchart illustrating dynamic update of $P H G$. 
sPHG and hitFlag are reset at the start of every interval. For every hit to a cache line 1 of an application $i$, if $H G_{l}>s P H G_{i}$, then $s P H G_{i}$ is set to $H_{1}$. At the end of the interval, $s P H G_{i}$ stores the maximum hit-gap of the application. If hitFlag ${ }_{i}$ is set, $P H G_{i}$ for the next interval is set to $s P H G_{i}+1$. If the hitFlag $i$ is reset, the application had no hits during the interval. In case of no hit, we provide application with maximum time in the cache for the next interval, i.e., $P H G=M A X$, assuming insufficient $P H G$. If the status of the hitFlag continues to remain reset, it means that the application has thrashing behavior, and so $s P H G$ is reset. To make sure such application is given opportunity to remain in the cache if its behavior changes, $P H G$ alternates between 0 and $M A X / 2$. Note that, any hit in the monitor or the normal cache set can change the value of $s P H G$ as long as the hit-gap of the current hit is greater than the present value of $s P H G$. Further, in the absence of monitor sets in the $P H G$ computation, the chances of the value of $s P H G$ being larger than $P H G$ would be less as all cache lines with $H G$ $=P H G$ are evicted.

Victim Selection : The most appropriate victim candidate will be a cache line that has been in the cache for its predicted-hit-gap time. It is not always necessary to find such a cache line and hence victim candidate that has the least time left in cache is chosen. As maximum time in the cache for a cache line $l$ of an application $i$ is when $H G_{l}=P H G_{i}$, the cache line with the minimum predicted life-time left in the cache will be the one with $\min \left(P H G_{i}-H G_{l}\right)$. Hence on a cache miss, the victim in the absence of an invalid or expired cache line is the cache line with $\min \left(P H G_{i}-H G_{l}\right)$. A victim with $\min \left(P H G_{i}-H G_{l}\right)$ is found from each core and then a suitable candidate is selected among them. This two-step procedure can be reduced to single step if we keep a additional pointer that always points to the most eligible candidate during the individual core victim search. The pointer to victim candidate will be modified based on the set's application wise-access recency information. Hence, the victim is a cache line that has minimum life in the cache from a core, which is least recently used. The worst case complexity of the victim selection logic is $O(k)$, where $k$ is the associativity of the cache (similar to TA-DRRIP). Figure 6 provides the flow of the victim selection logic.

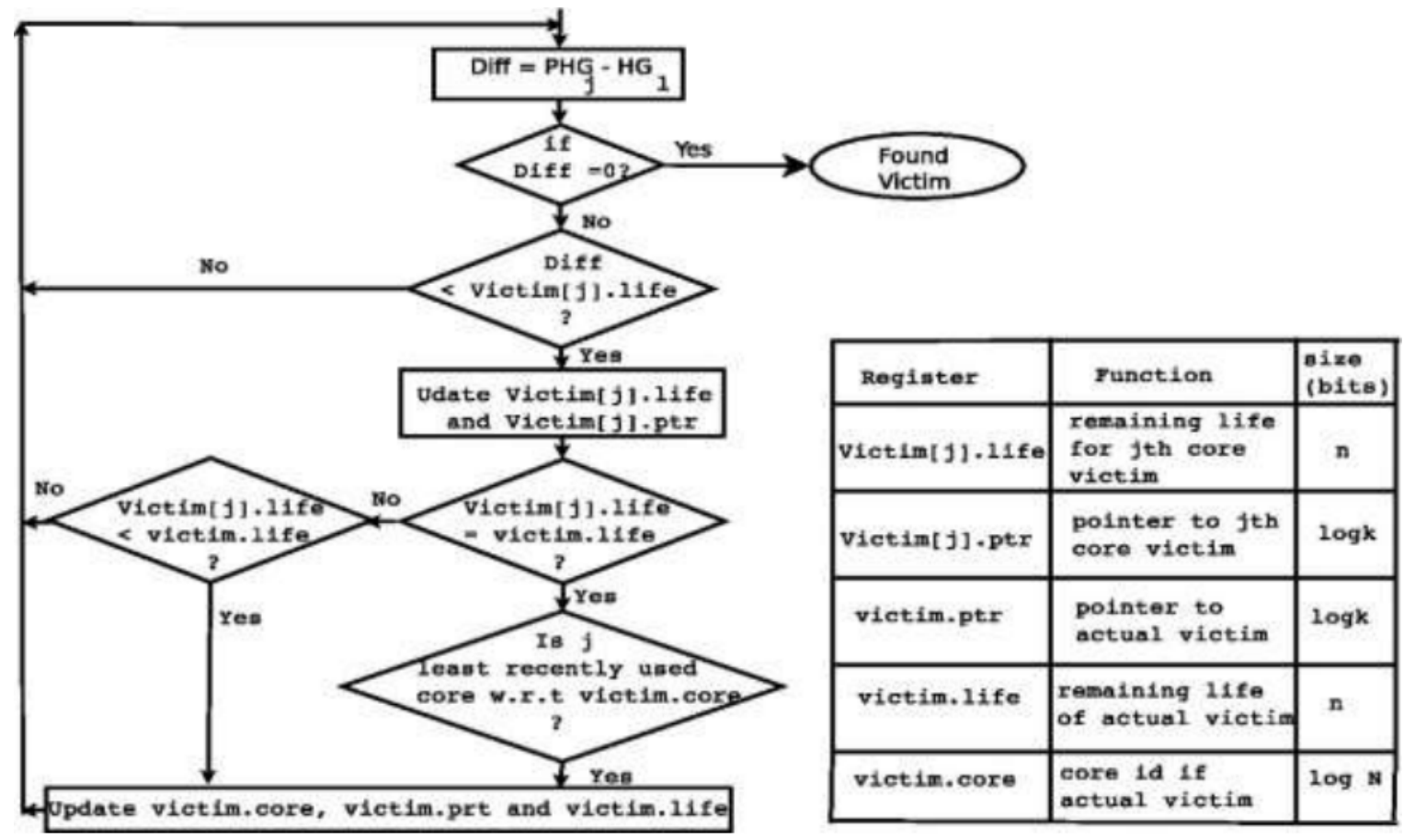

Figure 6: Victim Selection flow that will loop for associativity number of times in the worst case. 


\begin{tabular}{|l|l|}
\hline 164.gzip & 429.met \\
168.wupwise & 435.gromacs \\
171.swim & 437.leslic3d \\
172.mgrid & 444.namd \\
173.applu & 450.soplex \\
175.vpr & 454.calculix \\
177.mesa & 456.hmmer \\
178.galgel & 458.sjeng \\
179.art & 459.GemsFDTD \\
186.crafty & 462.libquantum \\
193.fma3d 5 & 464.h264ref \\
300.twolf & 470.lbm \\
401.brip2 & \\
\hline
\end{tabular}

\begin{tabular}{|l|l|}
\hline IL1 cahes & $\begin{array}{l}\text { 32KB, 64B, 4-way, 1 cycle, 1W and } \\
\text { 2R ports, LRU }\end{array}$ \\
\hline DL1 caches & $\begin{array}{l}\text { 32KB, 64B, 4-way, 1 cycle, 1W and } \\
\text { 2R ports, LRU }\end{array}$ \\
\hline L2 cache & $\begin{array}{l}\text { 256KB, 64B, 8-way, 10 cycles, } \\
\text { private, LRU }\end{array}$ \\
\hline LLC & $\begin{array}{l}\text { 1MB per-core, 64B, 16-way, 35 cycle, } \\
\text { shared, non-inclusive }\end{array}$ \\
\hline $\begin{array}{l}\text { Main } \\
\text { memory }\end{array}$ & 200 cycles \\
\hline
\end{tabular}

Table 1 SPEC CPU 2000 and 2006 benchmarks Table 2 Memory hierarchy of the simulated system.

This can be implemented using a couple of n-bit comparators, an n-bit subtractor and few registers, which will be controlled by an associated control logic. The victim selection procedure is not in the critical path and hence, the search involved in the algorithm does not affect the system performance.

When the LLC encounters scan access pattern, the corresponding cache lines become victims earlier by virtue of the application based $H G$ counter modification in both aACR and mACR techniques. Such scan patterns may not experience cache hits due to which cache lines of such applications are predicted to have smaller life-time in the cache. Hence, the ACR policy is scan resistant irrespective of the length of the scan chain unlike the SRRIP. The ACR policy is aware of access recency and so can perform well for the LRU-friendly applications. Hence in contrast to the TA-DRRIP, the ACR policy can perform well for applications with LRU-friendly or scan access patterns.

\section{EXPERIMENTAL SETUP}

Table 2 gives the details of the system configuration used in experimentation. The simulation setup consists of 3-level of cache having private L1 and L2 caches with a shared LLC. The basic system configuration used for evaluations is a 4-core system with one hardware thread per core. Multi-programmed workloads are executed on gem5 simulator [21]. Applications are fast-forwarded for 900 million instructions and then warmed up for next 100 million instructions. The statistics are recorded for the next 1 billion instructions for each application.

Workload Generation : The workloads consists of different benchmarks from SPEC CPU 2000 and 2006 suites [8] (refer to Table 1) compiled for ALPHA ISA. All the enchmarks are executed using reference inputs. Workloads with different mix of low (L) and high (H) MPKI values are considered for evaluation. Applications with MPKI $>3$ are categorized as $\mathrm{H}$ and others are categorized as L. The workload mixes are then grouped based on the number of low and high MPKI applications. We have $5((\# \mathrm{~L} ; \# \mathrm{H})=\mathrm{f}(4 ; 0) ;(3 ; 1) ;(2 ; 2) ;(1 ; 3) ;(0 ; 4) \mathrm{g})$ categories of workloads for 4-core system. About 20 mixes are considered for each class of workloads allowing repetition of benchmarks.

\section{RESULTS AND ANALYSIS}

We compare the ACR policy with LRU, TA-DRRIP [4] and PDP [9] based replacement techniques. We consider the feedback based TA-DRRIP [4] with 2N SDMs, where $\mathrm{N}$ is the number of cores, with 32 cache sets each to learn the insertion decision for each application. A 10-bit dedicated PSEL counter decides the core-wise insertion policy. We choose the parameters for the TA-DRRIP based on the optimum values discussed in [4]. 
The values of various parameters for the implementation of the PDP are as follows : maximum measured distance $d_{\max }=256$, Step counter for reuse distance counter array $S_{c}=$ 16, Distance Step for remaining protecting distance update $S_{d}=32$, and number of bits per cache line $\mathrm{n}_{\mathrm{c}}=3$. These choices of parameters for the PDP are according to [9] and lead to similar space overhead for the PDP and the ACR policy (without considering the protecting distance (PD) calculation logic in PDP). Since the evaluation framework considers noninclusive LLC, the bypass version of victim selection logic of the PDP is implemented to ensure fair comparison.

ACR technique considers 12-bit miss Counter to determine each phase of execution. Prior works $[3,17]$ have shown that 32 sets are sufficient to estimate the cache performance and so the implementation of the ACR policy uses 32 monitor sets. aACR and mACR techniques use $H G$ counters of width 4- and 3-bits, respectively. The evaluation framework considers the LLC with the LRU policy as the base-case.

\subsection{Effect on System Performance}

We evaluate the performance of multiple applications that are executed concurrently using weighted speed-up $\left(W S=\sum_{i} \frac{I P C_{i}^{\text {alone }}}{I P C_{i}^{\text {shared }}}\right)$ and harmonic speedup $\left(H S=N / \sum_{i} \frac{I P C_{i}^{\text {alone }}}{I P C_{i}^{\text {shared }}}\right)$. Here $I P C_{i}^{\text {alone }}$ is the IPC of application $i$ when run alone in the multi-core system, $I P C_{i}^{\text {shared }}$ is the IPC of the application $i$ in multi-core system and $\mathrm{N}$ is the number of cores in the system.

Figure 7 and Figure 8 show performance improvement of the ACR policy for a 4-core system compared to LRU, TA-DRRIP and PDP based replacement techniques using WS and HS . The geometric mean of WS for the aACR policy as compared to LRU, TA-DRRIP and PDP techniques are $8.74 \%, 5.16 \%$ and $3.2 \%$, respectively. mACR policy achieves a speed-up of $10.64 \%, 7.06 \%$ and $5.2 \%$ over LRU, TA-DRRIP and PDP based replacement techniques, respectively. aACR policy achieves a $11.37 \%, 7.47 \%$ and $5.34 \%$ improvement in HS over LRU, TA-DRRIP and PDP based techniques, respectively. Similarly the mACR policy achieves $13.53 \%, 9.63 \%$ and $7.27 \%$ improvement in HS over LRU, TA-DRRIP and PDP based techniques, respectively.
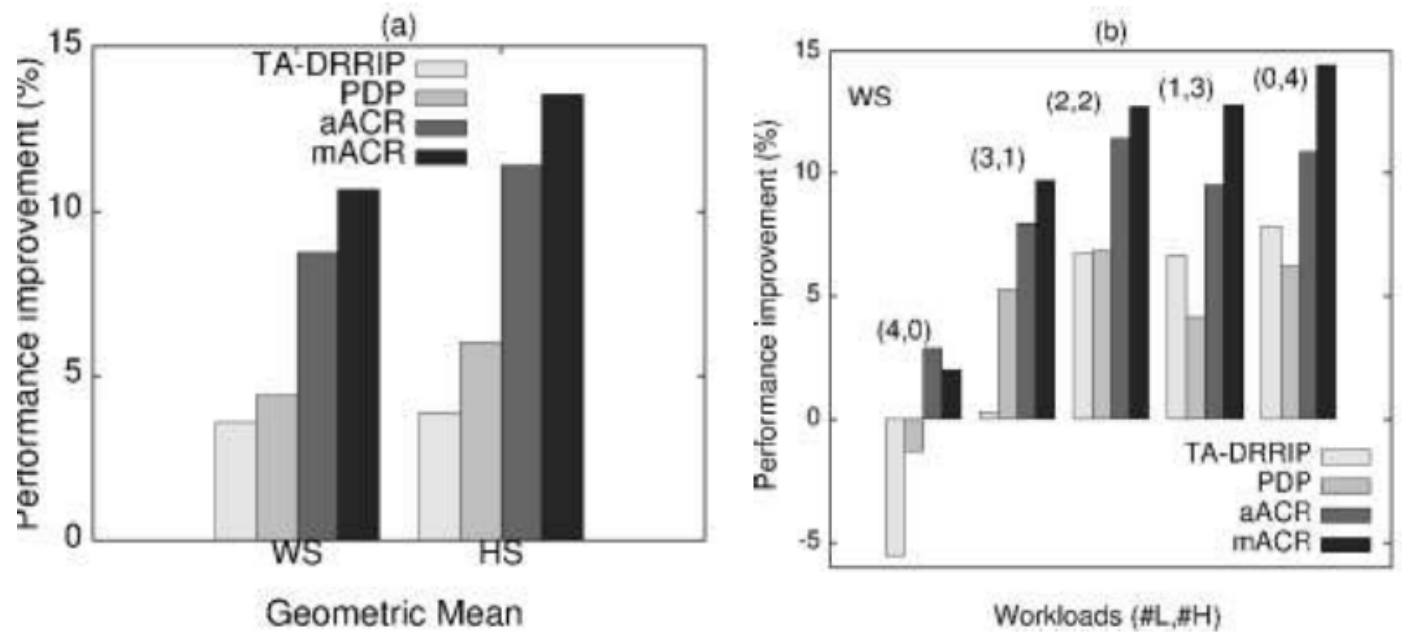

Figure 7 Performance comparison of the ACR policy for 4-core workloads normalized with respect to the TA-DRRIP in writeback LLC.

We analyze these results further in Figure 7 (b) that depicts the category-wise performance improvement in terms of WS . The different implementations of ACR policy outperforms LRU, TA-DRRIP and PDP techniques in all categories of workloads in a 4-core systems. The difference in performance improvement between the ACR policy and the TA- 
DRRIP is largest for $(4,0)$ workload category. This difference reduces as the number of $\mathrm{H}$ category applications increases in the workloads. Most of the applications in the L category are LRU-friendly and have low miss rate. As the modification of eviction priority chain for each application is similar to the LRU chain update in ACR policy, it improves the performance of workloads with the LRU-friendly patterns. On the other hand, in the absence of recency information in the TA-DRRIP, it degrades the performance of workloads with similar access patterns. Further, due to the dynamic update of length of the eviction priority chain based on the hit-gap in the ACR policy, applications with scan patterns will have shorter life-time in the cache due to small $P H G$ values. Hence both the implementations of the ACR policy are able to improve performance of workloads with both scan and LRU-friendly patterns. With increase in the number of high MPKI applications in workloads, the ACR policy, TA-DRRIP and PDP have improved performance. However, the performance of the ACR policy is still higher than TA-DRRIP and PDP techniques as it is access rate aware along with being access pattern aware. Even though the PDP is aware of reuse distance, its performance improvement for different categories of workloads is not comparable to the ACR policy. This is because the PDP tries to provide protection to cache lines with reuse distance as large as 256 accesses to a cache set. During this process, if the PDP does not find a victim, the incoming cache line may bypass the cache, which might not always work in favour of the application.

We now analyze the performance improvement in the ACR policy, by differentiating the contribution from the two changes made in the replacement priority chain. To study the effect of separating the individual application priority chain, we evaluate the static version of the ACR policy. For the static version of the ACR policy, we fix the maximum life-time of the cache lines for all the co-scheduled applications to be constant (15 in the case of aACR and 7 in the case of mACR). The static version of the aACR and the mACR provides a geometric mean speed-up of $3.8 \%$ and $6.6 \%$, respectively, over the LRU policy. This shows that isolating the eviction priority chains of individual applications is successful in reducing the harmful effects due to inter-core interference. LRU policy has a single priority chain for cache lines of all the co-scheduled applications and a cache miss for an application will update the status of all the cache lines. This can result in inter-core interference in the LRU policy. In TA-

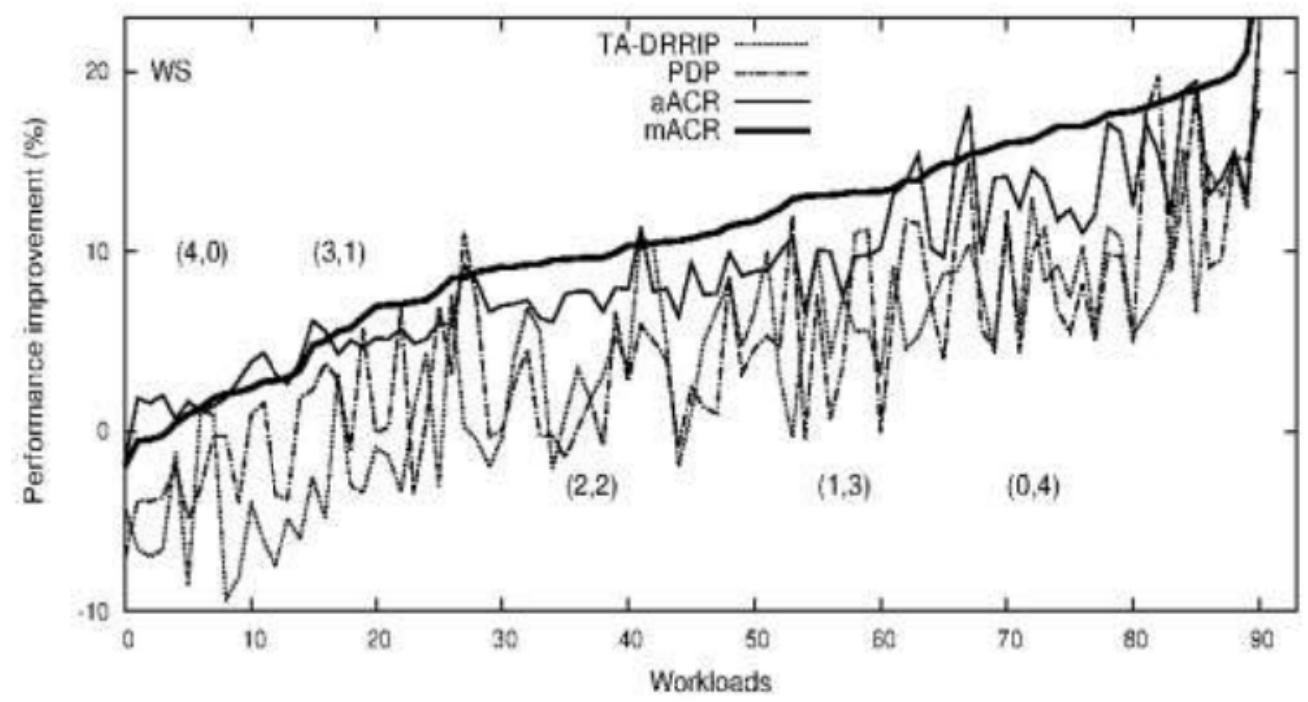

Figure 8 Effectiveness of the ACR policy for 4-core workloads normalized with respect to the LRU policy. 
DRRIP too, when there is no eligible victim (with $R R P V=3$ ), it updates the counter for all the cache lines that can have adverse effect on other co-scheduled applications. Even though the TA-DRRIP is able to prevent thrashing (by changing insertion policy using SDMs), it is not able to avoid inter-core interference among the cache-friendly applications. Further, the aACR and the mACR techniques dynamically adjust the length of different eviction priority chains and provide an additional improvement in speed-up by $4.94 \%$ and $4.04 \%$ respectively, in contrast to the LRU policy.

Table 3 Speedup $I P C_{i}^{\text {shared }} / I P C_{i}^{\text {alone }}$ of individual applications for workload \#90 (refer to Figure 8).

\begin{tabular}{|c|c|c|c|c|}
\hline Replacement policies & vpr & twolf & applu & lbm \\
\hline LRU & 0.4854 & 0.4663 & 0.9800 & 0.9942 \\
\hline TA-DRRIP & 0.7862 & 0.7331 & 0.9974 & 0.9957 \\
\hline PDP & 0.7664 & 0.6942 & 0.9884 & 0.9998 \\
\hline aACR & 0.8191 & 0.7779 & 0.9856 & 0.9947 \\
\hline mACR & 0.8739 & 0.8501 & 0.9806 & 0.9959 \\
\hline
\end{tabular}

Workload 90 in Figure 8 achieves the maximum speedup of $22.26 \%$ and $26.47 \%$, respectively for aACR and mACR implementations of the ACR policy. The corresponding workload is vpr-twolf-applu-lbm and it belongs to $(0,4)$ category. The split-up of individual applications that contribute to the speedup is provided in Table 3. The applications applu and $\mathrm{lbm}$ have no reuse (refer to Figure 2) due to which they have high miss rates (refer to Table 1). The other two applications $v p r$ and twolf have low miss rates and have higher locality in their accesses. The speedup for this workload with the TA-DRRIP is significant $(20.25 \%)$, which show that it is also able to identify the thrashing behavior of applu and $l \mathrm{bm}$. Both the TA-DRRIP and the ACR policy being scan resistant, successfully prevent the LLC pollution by applu and $l b m$. This results in improvement in performance of $v p r$ and twolf for both TADRRIP and ACR. Since TA-DRRIP searches for victim from left to right, it is not aware of access recency [22]. This may lead to inaccurate re-reference interval prediction. Recency in accesses influences the victim selection process in the ACR policy and cache lines of low miss rate, cache-friendly applications (such as $v p r$ and twolf) can remain in the cache for longer time based on their PHG. Due to this feature of the ACR policy, applications $v p r$ and twolf have larger speedup with the ACR policy as compared to the TA-DRRIP (refer to Table 3).

PDP gives a speedup of $17.8 \%$ for the same workload by keeping cache lines for protecting distance (PD) in the cache that is selected to maximize the overall hit rate in the LLC. But the PDP allows reuse distance as large as 256, which means that all cache lines from applications with large reuse distance remain in the LLC for longer time and pollute the LLC. Also, during a cache miss, the PDP inserts a cache line only if it finds a victim with no remaining life in the LLC. In the absence of such a candidate, the PDP bypasses the LLC, which can affect the application performance negatively. Decision of bypassing the LLC for an incoming cache line should be taken with utmost care since aggressive bypass of the LLC can increase the LLC miss rate. Since $v p r$ and twolf have good locality of reference, the PDP provides them with larger PD values to maximize the LLC hit rate. This can result in bypass of larger number of cache lines from these applications, limiting the performance improvement that can be achieved in them. Table 4 reiterates this by providing the average $P D s / P H G s$ and individual performance improvements by the PDP and the aACR policy for few workloads. Since the PDP keeps track of the reuse distance at a granularity of $S_{c}=16$ to reduce the area overhead, the PD can have values that are multiple of $S_{c}$. 
Table 4: Comparison of the PDP and the aACR policy for subset of workloads. Here, $\mathrm{P}$ denotes $P D$ or $P H G$ and $\mathrm{S}$ is individual speedup $\left(I P C_{i}^{\text {shared }} / I P C_{i}^{\text {alone }}\right)$.

\begin{tabular}{|c|c|c|c|c|c|c|c|c|c|}
\hline \multirow{2}{*}{ Workload (\#) } & \multirow{2}{*}{ Policy } & \multicolumn{2}{|c|}{ core 0} & \multicolumn{2}{|c|}{ core I } & \multicolumn{2}{|c|}{ core 2} & \multicolumn{2}{|c|}{ core 3} \\
\hline & & $P$ & $s$ & $\mathbf{P}$ & $\mathrm{s}$ & $P$ & s & $P$ & $\mathrm{~s}$ \\
\hline \multirow{2}{*}{$\begin{array}{c}175-300-173-168 \\
(90)\end{array}$} & PDP & 181.61 & 0.76 & 176.78 & 0.69 & 16.12 & 0.98 & 16 & 0.99 \\
\hline & $\mathrm{AACR}$ & 14.87 & 0.81 & 14.91 & 0.77 & 7.20 & 0.98 & 3.21 & 0.99 \\
\hline \multirow{2}{*}{$\begin{array}{c}164-456-459-454 \\
\text { (9) }\end{array}$} & PDP & 18.66 & 0.98 & 52.14 & 0.95 & 26.56 & 0.99 & 16.66 & 0.96 \\
\hline & AACR & 6.65 & 0.99 & 9.82 & 0.99 & 353 & 0.99 & 7.37 & 0.96 \\
\hline \multirow{2}{*}{$\begin{array}{c}172-171-400-175 \\
\text { (50) }\end{array}$} & & 16.7 & 0.85 & 16.39 & 0.99 & 68.82 & 0.81 & 152.98 & 0.77 \\
\hline & $\mathrm{aACR}$ & 3.74 & 0.96 & 4.45 & 0.99 & 70.53 & 0.84 & 1499 & 0.82 \\
\hline \multirow{2}{*}{$\begin{array}{c}164-178-456-459 \\
\text { (28) }\end{array}$} & PDP & 16.19 & 0.99 & 49.29 & 0.98 & 119.23 & 0.97 & 55.05 & 0.99 \\
\hline & AACR & 4.6 & 0.99 & 5.6 & 0.89 & 5.06 & 0.99 & 9.17 & 0.99 \\
\hline
\end{tabular}

PD of an application will be larger than or equal to the actual reuse distance of the application. Rounding of the reuse distance to larger value causes the PDP to hold cache lines in the cache for longer time than necessary. This can also result in additional misses in the LLC due to the increase in the LLC bypasses. The entries in Table 4 for workloads 9 and 50 confirm this phenomenon. The ACR policy handles such applications by providing the maximum $P H G$ values (restricted to 15 or 7 ) for them and not bypassing the LLC for any incoming cache line. This improves the overall performance of the system by better management of the shared LLC and confirms the observation in Section 2 that limiting the reuse distance to reasonable value provides significant number of cache hits for the LLC rereferences. An exception to this behaviour is seen in a couple of workloads $(28,82)$ where, the PDP outperforms the aACR and the mACR implementations of ACR policy marginally. In these cases, the PDP has calculated reasonable PD for each application (refer to Table 4) that aids in performance improvement in the corresponding applications without polluting the cache.

The evaluation shows that both aACR and mACR implementations of ACR policy are successful in improving system performance as compared to the existing techniques. Our policy works well for workloads that have LRU friendly and scan access patterns.

\subsection{Sensitivity to the Width of $H G$ Counter}

Figure 9 provides the effect of the width of the $H G$ counter on the performance of a 4-core system for a subset of workloads. Performance of the aACR policy improves with increase in the number of bits in the $H G$ counter from 2 bits to 5 bits. Increase in the number of bits for the $H G$ counter provides better control on the length of the eviction priority chains. Figure 9 shows that increasing the width of the $H G$ counter beyond 5-bits does not provide any further gain. This is because the cache has to retain the cache lines for more than 64 accesses to the corresponding cache set and retaining the cache lines for this long time may lead to cache pollution and degradation in the system performance. Thus, we consider 4-bit $H G$ counter for the aACR policy, as it provides significant performance improvement without incurring much hardware overhead. The study with variation of the $H G$ counter width for the mACR policy reveals that 3-bit counter is sufficient for the mACR policy implementation.

\subsection{Hardware Overhead}

For a 'k-way' set associative cache, the LRU policy and the TA-DRRIP have an overhead of $k \log k$ and $2 k$ bits per cache set, respectively. In addition, the TA-DRRIP has a 10-bit PSEL counter per-core to support SDMs. PDP has an overhead of $k[3$ (for RPD) $+\log C$ (for core id) ] $+C X 5$ (to use Distance Step $\mathrm{S}_{\mathrm{d}}=32$ ), where $C$ is the number of cores in the system. 


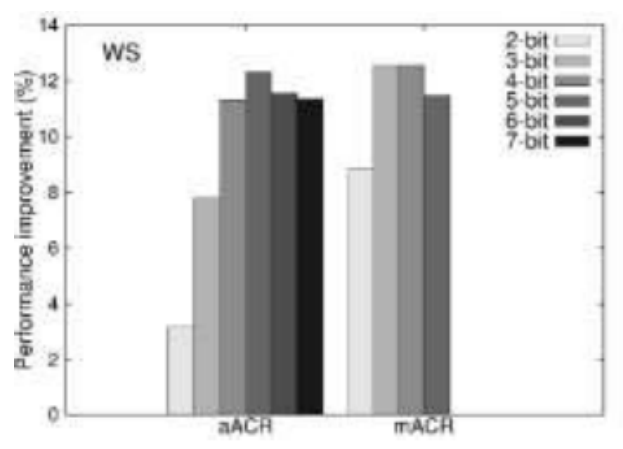

\begin{tabular}{|l|c|c|}
\hline $\begin{array}{l}\text { Replacement } \\
\text { Policy }\end{array}$ & $\begin{array}{c}\text { Bits per } \\
\text { Cache set }\end{array}$ & $\begin{array}{c}\text { \%Overhead w.r.t. } \\
\text { LLC size }\end{array}$ \\
\hline LRU & $16 * \log 16$ & 0.78 \\
\hline TA-DRRIP & $16 * 2$ & 0.39 \\
\hline PDP & $16 *(3+2)+4 * 5$ & 1.22 \\
\hline aACR & $16 *(4+2)+4 \log 4$ & 1.2 \\
\hline mACR & $16 *(3+2)+4 \log 4$ & 1.07 \\
\hline
\end{tabular}

Figure 9 Effect of the width of $H G$

Table 5 Overhead of replacement policies for 4-core

counter on performance of ACR policy. system with 4MB LLC used in result analysis.

Apart from this, the PDP has a reuse distance sampler, reuse distance counter array and a protecting distance compute logic which, helps the system to dynamically learn the reuse history. The ACR policy has an overhead of $k[\log k$ (for the $H G$ counters) $+\log C$ (for core id) ] $+C \log C$ (to implement core-wise LRU), where $C$ is the number of cores in the system. In addition to this, the ACR policy has two k-bit register for $P H G$ and $s P H G$, and one 1-bit register for hitFlag per application and a single 12-bit missCounter. The value of $\mathrm{k}$ for the aACR and the mACR implementations is 4 and 3, respectively. Table 5 shows the overhead in the implementation of LRU, TA-DRRIP, PDP, aACR and mACR replacement techniques for a 4-core system with 4MB LLC (used for the performance analysis in Section 5.1).

Though the ACR policy incurs larger hardware overhead than that of the TA-DRRIP, it achieves significant performance improvement in 4-core systems. Hence it can be used in systems where performance is critical and hardware overhead is not a constraint. The hardware overhead for implementing reuse distance sampler, reuse distance counter array and special purpose processor for the PD logic computing in the PDP technique is not added to the overhead calculation in Table 5. Tracking only the maximum reuse distance for each application (as opposed to finding the reuse distance that maximizes hits in the PDP) has greatly simplified the logic required for tracking the application awareness in the ACR policy. Even with a simple method of tracking reuse distance, the ACR policy achieves significant performance improvement over the PDP.

Victim selection procedure for our technique involves comparing the counter values to identify the victim candidate. This procedure is similar to that of the TA-DRRIP. Since the victim selection is in parallel to the memory access, it does not increase the critical path to the processor. For a cache miss, the baseline LRU policy updates the priorities of all the cache lines in the LLC. In case of a cache hit, the LRU policy may update the status of all the cache lines in the LLC based on the position of the cache line in the LRU chain. On the other hand, during a cache hit the TA-DRRIP updates the priority of only the hit cache line. For a miss at the LLC, the TA-DRRIP can modify the priorities of all the cache lines multiple times based on the availability of the victim. aACR policy is similar to the LRU policy while the mACR policy works like the TA-DRRIP. This process of updating replacement chain is similar for the PDP and the aACR policy as both of them measure the reuse distance based on accesses to set and hence cache line status is modified for every access. But it should be noted that in PDP, aACR and mACR, the priority modification occurs only for cache lines that belong to the accessing application. Among aACR and mACR implementations, mACR technique has an advantage due to the fact that the priorities of all the cache lines that belong to the accessing application could be updated only during a miss to the LLC. 

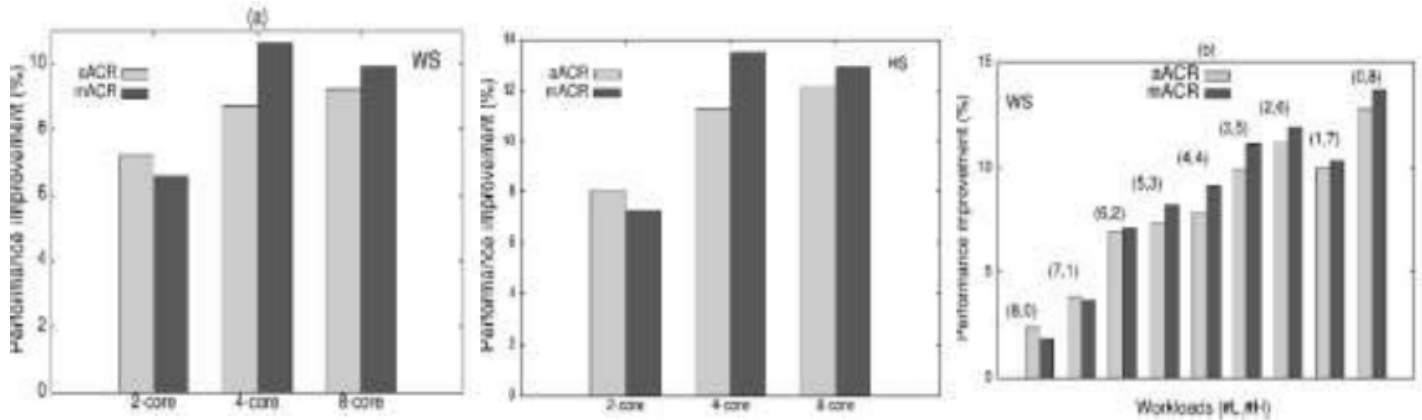

Figure 10 Scalability of ACR policy.

\subsection{Scalability}

This section analyzes the scalability of ACR policy by comparing performance for different categories of workload mixes in 2-core, 4-core and 8-core systems. Figure 10 shows the geometric mean of performance improvement in WS and HS obtained by the ACR policy for different system configurations. Different workload mixes for each multicore configuration were considered based on discussion in Section 4 . We have $3((\# \mathrm{~L}, \# \mathrm{H})=\{(2,0),(1,1),(0$, $2)\})$ and $9((\# \mathrm{~L}, \# \mathrm{H})=\{(8,0),(7,1),(6,2),(5,3),(4,4),(3,5),(2,6),(1,7) ;(0,8)\})$ categories of workloads for 2- and 8-core systems, respectively. Around 60 and 90 workloads for 2- and 8-core systems, respectively are considered for evaluation. An important observation from the figure is that across all the three systems there is significant speed-up over the baseline LRU policy. The geometric mean of speed-up of aACR policy for 2-,4- and 8 -core system is $7.1 \%, 8.7 \%$ and $9.1 \%$, respectively. mACR provides a speed-up of $6.5 \%$, $10.64 \%$ and $9.78 \%$ for $2-, 4-$ and 8 -core systems, respectively, over the LRU policy. The category-wise analysis of 8-core workloads in Figure 10(b) shows the trend similar to that seen in 4-core system (refer to Figure 7(b)).

\section{CONCLUSION}

A cache eviction policy based on reuse pattern of applications sharing the LLC is proposed for a multi-core system. As the proposed policy maintains separate eviction priority chains for applications sharing LLC, it prevents domination of high MPKI application over low MPKI application. It performs well in the presence of both LRU friendly and scan access patterns. Experiments on multi-core systems indicate that incorporating awareness of access and reuse distance during cache eviction is capable of decreasing the LLC miss rates and improving the LLC utilization. Evaluation of ACR policy using SPEC CPU 2000 and 2006 benchmark suites has shown to improve the performance with respect to LRU and other state of the art replacement policies (TA-DRRIP and PDP techniques). aACR policy and mACR policy outperforms the most recently proposed PDP by $3.2 \%$ and $5.2 \%$, respectively for a 4-core system. The maximum speed-up observed by mACR policy is $26.47 \%$ over the base line case for workload 90 in $(0,4)$ category. Further, evaluation of ACR policy techniques with 2-, 4and 8-core systems show significant performance improvement for all categories of workloads over the LRU replacement policy. In future work, we plan to apply fine grain control on the life of individual cache lines of applications at run-time along with the coarse grain maximum life constraints exerted by ACR policy 


\section{REFERENCES}

[1] L. Belady, A study of replacement algorithms for a virtual-storage computer, IBM Systems Journal 5 (2) (1966) 78-101.

[2] J. Jeong, M. Dubois, Cost-sensitive cache replacement algorithms, in: Proceedings of the 9th International Symposium on High-Performance Computer Architecture, HPCA '03, IEEE Computer Society, Washington, DC, USA. pp: 327-, 2003, pp. 327-.URL http://dl.acm.org/citation.cfm?id=822080.822804

[3] A. Jaleel, W. Hasenplaugh, M. Qureshi, J. Sebot, S. Steely, Jr., J. Emer, Adaptive insertion policies for managing shared caches, in: Proceedings of the International Conference on Parallel Architectures and Compilation Techniques (PACT), 2008, pp. 208-219.

[4] A. Jaleel, K. B. Theobald, S. C. Steely, Jr., J. Emer, High performance cache replacement using re-reference interval prediction (RRIP), in: Proceedings of the International Symposium on Computer Architecture (ISCA), 2010, pp. 60-71.

[5] C.-J. Wu, M. Martonosi, Adaptive timekeeping replacement: Fine-grained capacity management for shared $\mathrm{cmp}$ caches, ACM Transactions on Architecture and Code Optimization 8 (1) (2011) 3:1-3:26.

[6] Y. Xie, G. H. Loh, PIPP: Promotion/insertion pseudo-partitioning of multi-core shared caches, in: Proceedings of the International Symposium on Computer Architecture (ISCA), 2009, pp. 174-183.

[7] T. S. Warrier, B. Anupama, M. Mutyam, An application-aware cache replacement policy for last-level caches, in: Proceedings of the International Conference on Architecture of Computing Systems(ARCS), Springer Lecture Notes on Computer Science (LNCS), 2013, pp.207-219.

[8] SPEC CPU benchmark suite, http://www.spec.org.

[9] N. Duong, D. Zhao, T. Kim, R. Cammarota, M. Valero, A. V. Veidenbaum, Improving cache management policies using dynamic reuse distances, in: Proceedings of International Symposium on Microarchitecture (MICRO), 2012, pp. 389-400.

[10] M. Kharbutli, Y. Solihin, Counter-based cache replacement and bypassing algorithms, IEEE Transactions on Computers 57 (4) (2008) 433- 447.

[11] G. Keramidas, P. Petoumenos, S. Kaxiras, Cache replacement based on reuse distance prediction, in: Proceedings of the International Conference on Computer Design (ICCD), 2007, pp. 245-250.

[12] M. Feng, C. Tian, C. Lin, R. Gupta, Dynamic access distance driven cache replacement, ACM Transactions on Architecture and Code Optimization 8 (3) (2011) 14:1-14:30.

[13] P. Petoumenos, G. Keramidas, S. Kaxiras, Instruction-based reuse-distance prediction for e_ective cache management, in: Proceedings of the International Conference on Systems, Architectures, Modeling and Simulation (SAMOS), 2009, pp. 49-58.

[14] R. Manikantan, K. Rajan, R. Govindarajan, NUcache: An e_cient multicore cache organization based on next-use distance, in: Proceedings of the International Symposium on High Performance Computer Architecture (HPCA), 2011, pp. 243-253.

[15] C.-J. Wu, A. Jaleel, W. Hasenplaugh, M. Martonosi, S. C. Steely, Jr., J. Emer, Ship: signature-based hit predictor for high performance caching, in: Proceedings of the International Symposium on Microarchitecture (MICRO), 2011, pp. 430-441.

[16] D. A. Jim'enez, Insertion and promotion for tree-based pseudo lru last-level caches, in: Proceedings of the 46th Annual IEEE/ACM International Symposium on Microarchitecture, MICRO-46, 2013, pp. 284-296. 
[17] M. K. Qureshi, Y. N. Patt, Utility-based cache partitioning: A low-overhead, highperformance, runtime mechanism to partition shared caches, in: Proceedings of the International Symposium on Microarchitecture (MICRO), 2006, pp. 423-432.

[18] Y. Xie, G. Loh, Scalable shared-cache management by containing thrashing workloads, in: High Performance Embedded Architectures and Compilers, Lecture Notes in Computer Science (LNCS), 2010, pp. 262-276.

[19] D. Sanchez, C. Kozyrakis, Vantage: scalable and efficient fine-grain cache partitioning, in: Proceedings of International Symposium on Computer Architecture (ISCA), 2011, pp. $57-68$.

[20] R. Manikantan, K. Rajan, R. Govindarajan, Probabilistic shared cache management (prism), in: Proceedings of the International Symposium on Computer Architecture (ISCA), 2012, pp. 428-439.

[21] N. Binkert, B. Beckmann, G. Black, S. K. Reinhardt, A. Saidi, A. Basu, J. Hestness, D. R. Hower, T. Krishna, S. Sardashti, R. Sen, K. Sewell, M. Shoaib, N. Vaish, M. D. Hill, D. A. Wood, The GEM5 simulator, in: ACM SIGARCH Computer Architecture News, Vol. 39, 2011, pp. 1-7.

[22] X. Zhang, C. Li, H. Wang, D. Wang, A cache replacement policy using adaptive insertion and re-reference prediction, in: SBAC-PAD, 2010, pp. 95-102. 